\title{
Persistence, Relative Efficacy and Phytotoxicity of Lantana camara var. Aculeata (L.) Moldenke Leaf Crude Extracts in Hexane against Plutella xylostella L. in Cruciferous Vegetables
}

\author{
G. Thanavendan* and J.S. Kennedy \\ Department of Agricultural Entomology, Tamil Nadu Agricultural University \\ Coimbatore, Tamil Nadu, India \\ *Corresponding author
}

\section{A B S T R A C T}

\begin{tabular}{|l|}
\hline Ke y w or d s \\
Crude extracts, \\
Soxhlet \\
Extraction, \\
Persistent toxicity, \\
Phytotoxicity, \\
Brassicace \\
\hline Article Info \\
\hline Accepted: \\
29 May 2017 \\
Available Online: \\
10 June 2017 \\
\hline \hline
\end{tabular}

The present investigation was carried out to evaluate the phytochemicals from hexane extracts of wild sage, Lantana camara var. aculeata (L.) Moldenke. Out of the six concentrations tested against diamondback moth larvae on cauliflower, 6 , $8,10 \%$ were found to be more persistent than 1 and $2 \%$ against 2,3 and 4 th instar of DBM larvae. The order of efficacy of different treatments was hexane crude extracts $10 \%>8 \%>6 \%>4 \%>2 \%>1 \%$. However, the highest PT values to the extent of 7 days was observed in 6 per cent, when applied against second, third and fourth instar larvae of DBM on cauliflower and followed by 4 per cent upto 7 days without phytotoxicity. Moreover, 8 and $10 \%$ concentrations gave significant mortality but it caused phytotoxicity from 5 th days after spraying in cabbage and cauliflower. The effective biological action of the phytochemicals against the larvae varied with different instars. The crude extracts were biologically effective for 7 to14th day after treatment against second instars and their index of persistent toxicity was higher than that of third and fourth in stars. Phytotoxicity (\%) of crude extracts of 8 and $10 \%$ concentrations caused leaf tip injury as observed in both cauliflower (33.00 and $49.50 \%$ ) and cabbage (2.0 and 9.0) potted plants.

\section{Introduction}

Vegetable crops cover $1.1 \%$ of agriculture land worldwide (FAOSTAT, 2011) and can be particularly valuable sources of nutrition and income to small hold farmers. Members of the Brassicae group of vegetables are cultivated throughout the world. China tops the world rankings for production of cabbage and other Brassicas, with total of 31,750 million tonnes in 2011 (FAOSTAT, 2011). During 2013-2014, India produced 162.19 million tonnes of vegetables and exported worth of Rs. 5462.93 crores (Indian Horticulture Database, 2013). In Tamil Nadu, Cole vegetable occupies an area of 24000 and 9500 ha with an annual production of 130.42 and 209.17 MT and productivity is 50.00 and $22.00 \mathrm{t}$ ha-1 of cabbage and cauliflower respectively.

The important cosmopolitan insect pest of Brassica crop are Plutella xylostella L. (Sarfraz and Keddie, 2005; Liu et al., 2014). 
Systemic insecticides, which are usually applied in the early stages of plant growth, are often persistent within plants and can also have a harmful effect on some other beneficial insects, notably pollinators (Johnson et al., 2009). Overuse of pesticides can result in many ecological problems including, resistance, secondary pest outbreaks and pest resurgence due to a reduction in natural enemies (DeBach, 1974; Cloyd, 2012). Diamondback moth, P. xylostella a notorious insect known to developed resistance against most of the commercially available insecticides and other insects affecting vegetable crops (Krishnamoorthy, 2002). Pesticides at sub lethal concentrations have a strong impact on insects physiologically and behaviourally (Haynes, 1988). Owing to its ability to develop resistance to many conventional insecticides, the use of new insecticides which have low effects on other non-target organisms can be effective and helpful.

High value cash crops for industrial use (e.g. cotton) and human consumption (e.g. vegetables) have been areas, where problems associated with overuse of pesticides have been particularly manifest in crops (Zhao et al., 2002). One such example is control of the diamondback moth, P. xylostella on brassica crops (Sayyed et al., 2004; Badii et al., 2013; Furlong et al., 2013). The botanical insecticide mode of action may be contact, stomach or fumigant and the persistence of a botanical crude extracts in a given environment will determine the frequency of sprays required. Generally, effective period of an insecticide is evaluated by exposing the insect species to particular concentration of botanical insecticide. We have evaluated hexane crude extracts under pot culture experiments during 2015-16 against P. xylostella larvae and test the phytotoxicity of pot cultured 6 week old aged plants.

Materials and Methods
Mass culturing of diamondback moth, Plutella xylostella L. larvae

Culture of P. xylostella was initiated at Insectary, Department of Agricultural Entomology, TNAU, Coimbatore. DBM larvae were collected from farmer's field and reared on cauliflower leaves in the laboratory. Larval rearing was carried out in cages with the size of $30 \times 30 \times 30 \mathrm{~cm}$. The first instar larvae hatched in about 3 to 4 days were initially fed by mining into the mustard leaves and later on the entire leaves. For second instar larvae, tender cauliflower leaves were provided as feed material and larvae migrated to cauliflower leaves within a day and the larvae were provided with fresh leaves every day. To meet the daily requirement of leaves, cauliflower plants were grown continuously in pots and field. The larval duration of development from 1st to 4th instar larvae stage lasted for 3, 4, 5, and 6 days respectively (Harcourt, 1954) with the fourth larval instar having $1 \mathrm{~cm}$ in length and pupation mostly occurred on the lower surfaces of the leaves.

\section{Collection of test plant materials}

Aerial parts of the test plant L. camara were collected from Thondamuthur and Aalandurai block of Coimbatore district of Tamil Nadu. The samples were air-dried for 15-20 days under shade. After complete shade drying, the plant parts were pulverized into powder with the help of motor grinder.

\section{Extract preparation- Soxhlet Apparatus}

To extract more active principle, plant material was subjected to Soxhlet extraction (Sukthamrong et al., 1981; Sharma and Gupta, 2009). Known amount (75g thimple-1) of plant material of solvent was filled into the Soxhlet apparatus. A cotton plug was used at the place of thimble to stop the entry of the crude material into the siphoning tube. The 
required organic solvents were filled up five times more than total amount of the sample material into the flask of the apparatus. The apparatus was then connected with the water supply to the condenser. The temperature of the heating mantle was maintained according to the boiling point of respective solvents. The process was carried out for $24 \mathrm{~h}$ for each sample. Pooled extracts were filtered using a whatman filter paper no.1.and concentrated by rotary evaporation at $40{ }^{\circ} \mathrm{C}$. After drying in desiccator, crude extracts were weighed, stored in stock vials and kept in refrigerator $\left(4^{\circ} \mathrm{C}\right)$ for further use.

\section{Evaluation of Persistent toxicity}

Pot culture experiments were conducted in order to assess the persistent toxicity of Lantana crude extract as foliar application against diamondback moth larvae as per Mohamad and Ismail (1988) and Liu et al., (2003). The crude extracts were applied at seven different times to have the residual ages viz., 1, 3, 5, 7, 10, 14, 17 and 21 days after treatment (DAT) using hand atomizer spray equipped with delivery rate of $100 \mathrm{ml}$ per plant. Bioassays were initiated $3 \mathrm{~h}$ after treatment and single potted plant was used in each treatment. Each treated plant was placed in a large insect rearing cage and released ten numbers of larvae in each instar.

\section{Evaluation of phytotoxicity}

\section{Cauliflower and cabbage}

A pot culture experiment was conducted to determine the phytotoxicity, if any caused by Lantana crude extracts on cauliflower and cabbage at Coimbatore during 20142015.The experiment was conducted in CRD in a pot culture with three replications using hybrid of cauliflower and cabbage. The treatments include concentration of 1, 2, 4, 6, 8 and 10 per cent of Lantana crude extracts and negative control of solvents and water (1:1) used.

The plants were observed on $1,3,5,7,10,14$ and 21 DAT for the phytotoxic symptoms such as leaf tip injury, wilting, necrosis, vein clearing, epinasty and hyponasty. The extent of phytotoxicity was recorded based on the scale prescribed by Central Insecticide Board and Registration Committee (C.I.B and R.C).

\section{Method of assessment}

The per cent leaf injury was calculated using the following formula,

Total grade points

Per cent leaf injury = ----------------- x 100 Maximum grade $x$ Number of leaves observed

\section{Data analysis}

To test for the sub lethal effects of treatments on the demographic parameters of

P. xylostella, analysis of variance was performed. Significant difference was necessitated for the means to be separated by using LSD test (at $P=0.05$ ). All statistical tests were performed in IRRI Star version 2013.

\section{Results and Discussion}

\section{Persistent relative efficacy}

Efficacy of persistent toxicity against diamondback moth second instars showed greater susceptibility to the different concentrations tested for a longer period of time than the third and fourth in stars. The effect of the chemicals on the first and second instars remained significantly different until the seven and tenth day after spraying, when compared with the control. The crude extracts were least effective on the third and fourth 
instars after 10 days after spraying.

\section{Second instar}

Lantana crude extracts of all concentration were more biologically effective than the control on the second instar until 14 days after field spraying (Table 1) $10 \%$ concentration gave mortality of more than $60.82 \% .1,2$ and $4 \%$ were less effective mortality of 16.15 , 28.10 and $53.60 \%$ to the larvae respectively. $8 \%$ and $10 \%$ gave highest mortality only for 3 days after spraying (83.00; 96.43\%), whereas, the effect of 1 and $2 \%$ never attained $50 \%$ mortality.

\section{Third instar}

Effectiveness of the hexane crude extracts to the third instars per cent larval mortality is indicated (Table 2). 1, 2 and $4 \%$ concentrations showed only some degree of effectiveness to the third instar larvae. The effective persistence period was upto 10 days with highest PTI 559.90, 701.44 and 846.00 of 6,8 and $10 \%$ concentrations with mortality is $39.99,50.10$ and 60.43 respectively. However, this mortality was very much reduced compared to the second instars. The mortality period remained significantly different until 10 day after spraying when compared to that of the control.

\section{Fourth instar}

As regards to residual foliar toxicity in pot culture experiment have deposits of hexane crude extracts observation took different interval periods after application indicated per cent larval mortality recorded (Table 3 ). The toxicity recorded 7 days after crude extract application was reported to be the highest PTI was (710.24) in treatment with $10 \%$ $(50.73 \%)$, however it was on par in 8 and6\% $(47.81 ; 39.40 \%)$ with PTI was (669.34; 551.64). In the treatment 1,2and $4 \%$ consistently lower larval mortality was reported when taken at 7th DAT $(12.03,18.84$ and $28.71 \%$ ) larval mortality decreased. The persistent toxicity (PT) values ranged from 90.51 to 191.58 and effective concentrations of $1 \%$ neem oil persisted for six days but its persistence prolonged to nine days at 2, 3 and $4 \%$ respectively on green leaf hopper, Nephotettix virescens Distant in rice (Dash et al., 1995).

\section{Phytotoxicity of Lantana crude extracts on cauliflower and cabbage per cent damage}

All the treatments irrespective of the concentrations did not give inflict any phytotoxicity symptoms like injury to the leaf tip, wilting, necrosis, vein clearing, epinasty and hyponasty. However, the phytotoxicity of hexane crude extracts of 8 and $10 \%$ concentrations caused leaf tip injury were observed in both cauliflower $(33.00$ and $49.50 \%$ ) and cabbage (2.0 and 9.0) of potted plants. The crude extracts also evaluated physical compatibility test (Table 5.) of hexane crude extracts of Lantana leaves with other botanicals such as neem oil and NSKE before final field spray initiated. Upto 6\% concentration there is no leaf tip injury was noticed after treatments imposed and per cent damage is mentioned in Table 4.The aqueous solution (5.00 gL-1) with extract of Lantana leaves completely killed Eichhornia crassipes (Mart.) and drastically reduced the biomass of Microcystis aeruginosa Kutz. Within 7 days in freshwater systems, particularly in eutrophicated lakes in china (Kong et al., 2005). 
Table.1 Persistence toxicity of hexane crude extracts of Lantana leaves against $2^{\text {nd }}$ instar of Plutella xylostella L. at $24 \mathrm{~h}$ after exposure

\begin{tabular}{|c|c|c|c|c|c|c|c|c|c|c|}
\hline \multirow{2}{*}{$\begin{array}{l}\% \\
\text { Conc. }\end{array}$} & \multicolumn{10}{|c|}{${ }^{\wedge}$ Days after treatment - Mean per cent larval mortality* } \\
\hline & $\mathbf{0}$ & $\mathbf{1}$ & 3 & 5 & 7 & $\mathbf{1 0}$ & 14 & $\mathbf{T}$ & PTI & ORE \\
\hline 1.00 & $\begin{array}{l}0.12 \\
(1.99)^{\mathrm{b}}\end{array}$ & $\begin{array}{l}27.59 \\
(31.69)^{b c}\end{array}$ & $\begin{array}{l}41.38 \\
(40.04)^{b}\end{array}$ & $\begin{array}{l}27.59 \\
(31.69)^{\mathrm{c}}\end{array}$ & $\begin{array}{l}0.12 \\
(1.99)^{\mathrm{c}}\end{array}$ & $\begin{array}{l}0.12 \\
(1.99)^{\mathrm{d}}\end{array}$ & $\begin{array}{l}0.12 \\
(1.99)^{d}\end{array}$ & 16.15 & 80.77 & 6 \\
\hline 2.00 & $\begin{array}{l}0.12 \\
(1.99)^{b}\end{array}$ & $\begin{array}{l}20.69 \\
(27.06)^{b c}\end{array}$ & $\begin{array}{l}75.86 \\
(60.57)^{\mathrm{a}}\end{array}$ & $\begin{array}{l}58.62 \\
(49.96)^{b}\end{array}$ & $\begin{array}{l}10.00 \\
(18.43)^{\mathrm{c}}\end{array}$ & $\begin{array}{l}3.33 \\
(10.51)^{d}\end{array}$ & $\begin{array}{l}0.12 \\
(1.99)^{d}\end{array}$ & 28.10 & 281.03 & 5 \\
\hline 4.00 & $\begin{array}{l}0.12 \\
(1.99)^{b}\end{array}$ & $\begin{array}{l}20.00 \\
(26.57)^{\mathrm{c}}\end{array}$ & $\begin{array}{l}96.43 \\
(79.11)^{\mathrm{a}}\end{array}$ & $\begin{array}{l}89.66 \\
(71.24)^{\mathrm{a}}\end{array}$ & $\begin{array}{l}60.34 \\
(50.97)^{\mathrm{a}}\end{array}$ & $\begin{array}{l}44.83 \\
(42.03)^{\mathrm{c}}\end{array}$ & $\begin{array}{l}10.33 \\
(18.75)^{\mathrm{c}}\end{array}$ & 53.60 & 750.38 & 4 \\
\hline 6.00 & $\begin{array}{l}0.12 \\
(1.99)^{b}\end{array}$ & $\begin{array}{l}16.67 \\
(24.10)^{\mathrm{c}}\end{array}$ & $\begin{array}{l}82.14 \\
(65.00)^{b}\end{array}$ & $\begin{array}{l}75.86 \\
(60.57)^{b}\end{array}$ & $\begin{array}{l}54.33 \\
(47.48)^{\mathrm{b}}\end{array}$ & $\begin{array}{l}48.00 \\
(43.85)^{b}\end{array}$ & $\begin{array}{l}20.67 \\
(27.04)^{b}\end{array}$ & 55.45 & 776.35 & 3 \\
\hline 8.00 & $\begin{array}{l}6.67 \\
(14.97)^{\mathrm{a}}\end{array}$ & $\begin{array}{l}34.48 \\
(35.96)^{\mathrm{ab}}\end{array}$ & $\begin{array}{l}83.00 \\
(65.65)^{\mathrm{a}}\end{array}$ & $\begin{array}{l}93.10 \\
(74.77)^{\mathrm{a}}\end{array}$ & $\begin{array}{l}66.00 \\
(54.33)^{\mathrm{a}}\end{array}$ & $\begin{array}{l}53.00 \\
(46.72)^{\mathrm{ab}}\end{array}$ & $\begin{array}{l}32.00 \\
(34.45)^{\mathrm{a}}\end{array}$ & 60.26 & 843.69 & 2 \\
\hline 10.00 & $\begin{array}{l}6.67 \\
(14.97)^{\mathrm{a}}\end{array}$ & $\begin{array}{l}51.72 \\
(45.99)^{\mathrm{a}}\end{array}$ & $\begin{array}{l}96.43 \\
(79.11)^{\mathrm{a}}\end{array}$ & $\begin{array}{l}86.21 \\
(68.20)^{\mathrm{a}}\end{array}$ & $\begin{array}{l}70.00 \\
(56.79)^{\mathrm{a}}\end{array}$ & $\begin{array}{l}58.62 \\
(49.96)^{\mathrm{a}}\end{array}$ & $\begin{array}{l}37.00 \\
(37.46)^{\mathrm{a}}\end{array}$ & 60.82 & 851.50 & 1 \\
\hline$(\mathrm{S}: \mathrm{W})^{@}$ & $\begin{array}{l}0.12 \\
(1.99)^{\mathrm{b}}\end{array}$ & $\begin{array}{l}0.12 \\
(1.99)^{\mathrm{d}}\end{array}$ & $\begin{array}{l}3.33 \\
(10.51)^{c}\end{array}$ & $\begin{array}{l}3.33 \\
(10.51)^{d}\end{array}$ & $\begin{array}{l}3.33 \\
(10.51)^{\mathrm{c}}\end{array}$ & $\begin{array}{l}0.12 \\
(1.99)^{\mathrm{d}}\end{array}$ & $\begin{array}{l}0.12 \\
(1.99)^{\mathrm{d}}\end{array}$ & 1.73 & - & 7 \\
\hline $\mathrm{SE}(\mathrm{d})$ & 0.61 & 6.56 & 8.54 & 6.93 & 4.43 & 3.06 & 8.59 & - & - & - \\
\hline $\mathrm{CD}(0.05)$ & 1.29 & 4.06 & 12.61 & 4.85 & 9.51 & 6.57 & 11.84 & - & - & - \\
\hline
\end{tabular}

@ - Solvent water mixture (1:1); ^P - Period of persistence (days) PTI - Persistent Toxicity Index; T - Mean per cent mortality; ORE- Order of relative efficacy; *at days post-insecticidal treatment. Means in the same column bearing the same letter are not significantly different at P < 0.05 as determined by LSD. 
Table.2 Persistence toxicity of hexane crude extracts of Lantana leavesagainst $3^{\text {rd }}$ instar of Plutella xylostella L. at $24 \mathrm{~h}$ after exposure

\begin{tabular}{|c|c|c|c|c|c|c|c|c|c|c|}
\hline \multirow{2}{*}{$\begin{array}{l}\% \\
\text { Conc. }\end{array}$} & \multicolumn{10}{|c|}{${ }^{\wedge}$ Days after treatment - Mean per cent larval mortality* } \\
\hline & $\mathbf{0}$ & 1 & 3 & 5 & 7 & 10 & 14 & $\mathbf{T}$ & PTI & ORE \\
\hline 1.00 & $\begin{array}{l}0.12 \\
(1.99)^{\mathrm{c}}\end{array}$ & $\begin{array}{l}30.00 \\
(33.21)^{\mathrm{e}}\end{array}$ & $\begin{array}{l}30.00 \\
(33.21)^{f}\end{array}$ & $\begin{array}{l}27.59 \\
(31.69)^{f}\end{array}$ & $\begin{array}{l}10.34 \\
(18.76)^{\mathrm{f}}\end{array}$ & $\begin{array}{l}0.12 \\
(1.99)^{\mathrm{f}}\end{array}$ & $\begin{array}{l}0.12 \\
(1.99)^{\mathrm{f}}\end{array}$ & 14.04 & 70.21 & 6 \\
\hline 2.00 & $\begin{array}{l}0.12 \\
(1.99)^{\mathrm{c}}\end{array}$ & $\begin{array}{l}38.00 \\
(38.06)^{d}\end{array}$ & $\begin{array}{l}40.00 \\
(39.23)^{\mathrm{e}}\end{array}$ & $\begin{array}{l}40.00 \\
(39.23)^{\mathrm{e}}\end{array}$ & $\begin{array}{l}33.00 \\
(35.06)^{\mathrm{e}}\end{array}$ & $\begin{array}{l}10.34 \\
(18.76)^{\mathrm{e}}\end{array}$ & $\begin{array}{l}0.12 \\
(1.99)^{\mathrm{e}}\end{array}$ & 23.08 & 230.83 & 5 \\
\hline 4.00 & $\begin{array}{l}0.12 \\
(1.99)^{\mathrm{c}}\end{array}$ & $\begin{array}{l}43.00 \\
(40.98)^{c}\end{array}$ & $\begin{array}{l}45.00 \\
(42.13)^{\mathrm{d}}\end{array}$ & $\begin{array}{l}44.00 \\
(41.55)^{\mathrm{d}}\end{array}$ & $\begin{array}{l}44.83 \\
(42.03)^{\mathrm{d}}\end{array}$ & $\begin{array}{l}24.14 \\
(29.43)^{d}\end{array}$ & $\begin{array}{l}6.67 \\
(14.97)^{d}\end{array}$ & 29.68 & 415.52 & 4 \\
\hline 6.00 & $\begin{array}{l}0.12 \\
(1.99)^{\mathrm{c}}\end{array}$ & $\begin{array}{l}44.83 \\
(42.03)^{\mathrm{c}}\end{array}$ & $\begin{array}{l}53.00 \\
(46.72)^{\mathrm{c}}\end{array}$ & $\begin{array}{l}55.00 \\
(47.87)^{\mathrm{c}}\end{array}$ & $\begin{array}{l}48.00 \\
(43.85)^{\mathrm{c}}\end{array}$ & $\begin{array}{l}47.00 \\
(43.28)^{\mathrm{c}}\end{array}$ & $\begin{array}{l}32.00 \\
(34.45)^{\mathrm{c}}\end{array}$ & 39.99 & 559.90 & 3 \\
\hline 8.00 & $\begin{array}{l}6.67 \\
(14.97)^{b}\end{array}$ & $\begin{array}{l}51.72 \\
(45.99)^{\mathrm{b}}\end{array}$ & $\begin{array}{l}69.00 \\
(56.17)^{b}\end{array}$ & $\begin{array}{l}66.00 \\
(54.33)^{\mathrm{b}}\end{array}$ & $\begin{array}{l}65.00 \\
(53.73)^{b}\end{array}$ & $\begin{array}{l}55.33 \\
(48.06)^{b}\end{array}$ & $\begin{array}{l}37.00 \\
(37.46)^{b}\end{array}$ & 50.10 & 701.44 & 2 \\
\hline 10.00 & $\begin{array}{l}10.00 \\
(18.43)^{\mathrm{a}}\end{array}$ & $\begin{array}{l}55.00 \\
(47.87)^{\mathrm{a}}\end{array}$ & $\begin{array}{l}80.00 \\
(63.43)^{\mathrm{a}}\end{array}$ & $\begin{array}{l}82.00 \\
(64.90)^{\mathrm{a}}\end{array}$ & $\begin{array}{l}74.00 \\
(59.34)^{\mathrm{a}}\end{array}$ & $\begin{array}{l}78.00 \\
(62.03)^{\mathrm{a}}\end{array}$ & $\begin{array}{l}44.00 \\
(41.55)^{\mathrm{a}}\end{array}$ & 60.43 & 846.00 & 1 \\
\hline$(\mathrm{S}: \mathrm{W})^{@}$ & $\begin{array}{l}0.12 \\
(1.99)^{\mathrm{c}}\end{array}$ & $\begin{array}{l}3.33 \\
(10.51)^{f}\end{array}$ & $\begin{array}{l}0.12 \\
(1.99)^{\mathrm{g}}\end{array}$ & $\begin{array}{l}0.12 \\
(1.99)^{\mathrm{g}}\end{array}$ & $\begin{array}{l}0.12 \\
(1.99)^{\mathrm{g}}\end{array}$ & $\begin{array}{l}0.12 \\
(1.99)^{\mathrm{g}}\end{array}$ & $\begin{array}{l}0.12 \\
(1.99)^{\mathrm{g}}\end{array}$ & 0.58 & 0.00 & 7 \\
\hline $\mathrm{SE}(\mathrm{d})$ & 2.11 & 0.59 & 1.34 & 1.19 & 1.35 & 0.90 & 0.59 & - & - & - \\
\hline $\mathrm{CD}(0.05)$ & 4.53 & 1.27 & 2.88 & 2.57 & 2.89 & 1.95 & 1.27 & - & - & - \\
\hline
\end{tabular}

@ - Solvent water mixture (1:1); ^P - Period of persistence (days) PTI - Persistent Toxicity Index; T - Mean per cent mortality; ORE- Order of relative efficacy;

*at days post-insecticidal treatment. Means in the same column bearing the same letter are not significantly different at P < 0.05 as determined by LSD. 
Table.3 Persistence toxicity of hexane crude extracts of Lantana leavesagainst $4^{\text {th }}$ instar of Plutella xylostella L.at 24 h after exposure

\begin{tabular}{|c|c|c|c|c|c|c|c|c|c|c|}
\hline \multirow{2}{*}{$\begin{array}{l}\% \\
\text { Conc. }\end{array}$} & \multicolumn{10}{|c|}{${ }^{\wedge}$ Days after treatment - Mean per cent larval mortality* } \\
\hline & $\mathbf{0}$ & 1 & 3 & 5 & 7 & 10 & 14 & $\mathbf{T}$ & PTI & ORE \\
\hline 1.00 & $\begin{array}{l}0.12 \\
(1.99)\end{array}$ & $\begin{array}{l}20.00 \\
(26.57)^{\mathrm{e}}\end{array}$ & $\begin{array}{l}21.00 \\
(27.27)^{\mathrm{d}}\end{array}$ & $\begin{array}{l}21.00 \\
(27.27)^{\mathrm{f}}\end{array}$ & $\begin{array}{l}22.00 \\
(27.97)^{\mathrm{c}}\end{array}$ & $\begin{array}{l}0.12 \\
(1.99)^{\mathrm{e}}\end{array}$ & $\begin{array}{l}0.12 \\
(1.99)\end{array}$ & 12.03 & 84.24 & 6 \\
\hline 2.00 & $\begin{array}{l}0.12 \\
(1.99)\end{array}$ & $\begin{array}{l}27.00 \\
(31.31)^{d}\end{array}$ & $\begin{array}{l}27.67 \\
(31.74)^{b c}\end{array}$ & $\begin{array}{l}27.00 \\
(31.31)^{\mathrm{e}}\end{array}$ & $\begin{array}{l}27.00 \\
(31.31)^{b c}\end{array}$ & $\begin{array}{l}0.12 \\
(1.99)^{\mathrm{e}}\end{array}$ & $\begin{array}{l}0.12 \\
(1.99)\end{array}$ & 18.84 & 188.44 & 5 \\
\hline 4.00 & $\begin{array}{l}0.12 \\
(1.99)\end{array}$ & $\begin{array}{l}32.00 \\
(34.45)^{\mathrm{c}}\end{array}$ & $\begin{array}{l}35.00 \\
(36.27)^{b c}\end{array}$ & $\begin{array}{l}35.00 \\
(36.27)^{d}\end{array}$ & $\begin{array}{l}35.00 \\
(36.27)^{\mathrm{ab}}\end{array}$ & $\begin{array}{l}21.00 \\
(27.27)^{d}\end{array}$ & $\begin{array}{l}0.12 \\
(1.99)\end{array}$ & 28.71 & 401.96 & 4 \\
\hline 6.00 & $\begin{array}{l}0.12 \\
(1.99)\end{array}$ & $\begin{array}{l}46.00 \\
(42.71)^{b}\end{array}$ & $\begin{array}{l}48.00 \\
(43.85)^{b}\end{array}$ & $\begin{array}{l}48.00 \\
(43.85)^{\mathrm{c}}\end{array}$ & $\begin{array}{l}47.70 \\
(43.68)^{\mathrm{a}}\end{array}$ & $\begin{array}{l}30.00 \\
(33.21)^{\mathrm{c}}\end{array}$ & $\begin{array}{l}0.12 \\
(1.99)\end{array}$ & 39.40 & 551.64 & 3 \\
\hline 8.00 & $\begin{array}{l}0.12 \\
(1.99)\end{array}$ & $\begin{array}{l}57.00 \\
(49.02)^{b}\end{array}$ & $\begin{array}{l}59.00 \\
(50.18)^{\mathrm{a}}\end{array}$ & $\begin{array}{l}59.00 \\
(50.18)^{b}\end{array}$ & $\begin{array}{l}58.62 \\
(49.96)^{\mathrm{a}}\end{array}$ & $\begin{array}{l}37.93 \\
(38.02)^{b}\end{array}$ & $\begin{array}{l}0.12 \\
(1.99)\end{array}$ & 47.81 & 669.34 & 2 \\
\hline 10.00 & $\begin{array}{l}0.12 \\
(1.99)\end{array}$ & $\begin{array}{l}62.00 \\
(51.94)^{\mathrm{a}}\end{array}$ & $\begin{array}{l}64.00 \\
(53.13)^{\mathrm{a}}\end{array}$ & $\begin{array}{l}61.00 \\
(51.35)^{\mathrm{a}}\end{array}$ & $\begin{array}{l}62.00 \\
(51.94)^{\mathrm{a}}\end{array}$ & $\begin{array}{l}42.00 \\
(40.40)^{\mathrm{a}}\end{array}$ & $\begin{array}{l}0.12 \\
(1.99)\end{array}$ & 50.73 & 710.24 & 1 \\
\hline$(\mathrm{S}: \mathrm{W})^{@}$ & $\begin{array}{l}0.12 \\
(1.99)\end{array}$ & $\begin{array}{l}0.12 \\
(1.99)^{\mathrm{f}}\end{array}$ & $\begin{array}{l}0.12 \\
(1.99)^{\mathrm{e}}\end{array}$ & $\begin{array}{l}0.12 \\
(1.99)^{\mathrm{g}}\end{array}$ & $\begin{array}{l}0.12 \\
(1.99)^{\mathrm{d}}\end{array}$ & $\begin{array}{l}0.12 \\
(1.99)^{\mathrm{e}}\end{array}$ & $\begin{array}{l}0.12 \\
(1.99)\end{array}$ & 0.12 & 0.00 & 7 \\
\hline $\mathrm{SE}(\mathrm{d})$ & 0.00 & 1.18 & 1.58 & 0.88 & 0.80 & 1.19 & 0.00 & - & - & - \\
\hline $\mathrm{CD}(0.05)$ & 0.00 & 2.54 & 3.38 & 1.89 & 1.73 & 2.56 & 0.00 & - & - & - \\
\hline
\end{tabular}

@ - Solvent water mixture (1:1); ^P - Period of persistence (days) PTI - Persistent Toxicity Index; T - Mean per cent mortality; ORE- Order of relative efficacy;

*at days post-insecticidal treatment. Means in the same column bearing the same letter are not significantly different at P < 0.05 as determined by LSD. 
Table.4 Phytotoxicity of hexane crude extracts of Lantana leaves on cauliflower and cabbage pot culture experiment

\begin{tabular}{|c|c|c|c|c|c|c|c|c|c|c|c|}
\hline \multirow{3}{*}{$\begin{array}{l}\% \\
\text { Conc. } \\
\text { DAT }\end{array}$} & \multicolumn{11}{|c|}{ Cauliflower - Phytotoxicity rating ${ }^{\wedge}$} \\
\hline & \multicolumn{5}{|c|}{ Leaf tip injury \% } & \multirow{2}{*}{$\begin{array}{l}\text { Wiltin } \\
\text { g } \\
-\end{array}$} & \multirow{2}{*}{$\begin{array}{l}\begin{array}{l}\text { Vein } \\
\text { clearing }\end{array} \\
-\end{array}$} & \multirow{2}{*}{$\begin{array}{l}\text { Necrosi } \\
\text { S } \\
-\end{array}$} & \multirow{2}{*}{$\begin{array}{l}\text { Epinast } \\
\mathbf{y} \\
-\end{array}$} & \multirow{2}{*}{$\begin{array}{l}\text { Hyponasty } \\
-\end{array}$} & \multirow{2}{*}{$\begin{array}{l}\text { Mean \% } \\
\text { Damage } \\
-\end{array}$} \\
\hline & 5 & 7 & 10 & 14 & 21 & & & & & & \\
\hline 1.00 & - & - & - & - & - & - & - & - & - & - & - \\
\hline 2.00 & - & - & - & - & - & - & - & - & - & - & - \\
\hline 4.00 & - & - & - & - & - & - & - & - & - & - & - \\
\hline 6.00 & - & - & - & - & - & - & - & - & - & - & - \\
\hline 8.00 & 27.50 & 30.00 & 32.50 & 35.00 & 40.00 & - & - & - & - & - & 33.00 \\
\hline 10.00 & 37.50 & 47.50 & 47.50 & 55.00 & 60.00 & - & - & - & - & - & 49.50 \\
\hline$(\mathrm{S}: \mathrm{W})^{@}$ & 60.00 & 67.50 & 70.00 & 75.00 & 75.00 & - & - & - & - & - & 69.50 \\
\hline
\end{tabular}

Cabbage - Phytotoxicity rating ${ }^{\wedge}$

\begin{tabular}{|c|c|c|c|c|c|c|c|c|c|c|c|}
\hline DAT & 5 & 7 & 10 & 14 & 21 & - & - & - & - & - & - \\
\hline 1.00 & - & - & - & - & - & - & - & - & - & - & - \\
\hline 2.00 & - & - & - & - & - & - & - & - & - & - & - \\
\hline 4.00 & - & - & - & - & - & - & - & - & - & - & - \\
\hline 6.00 & - & - & - & - & - & - & - & - & - & - & - \\
\hline 8.00 & 1.00 & 1.50 & 2.50 & 2.50 & 2.50 & - & - & - & - & - & 2.00 \\
\hline 10.00 & 6.50 & 7.00 & 11.00 & 11.00 & 11.00 & - & - & - & - & - & 9.30 \\
\hline$(\mathrm{S}: \mathrm{W})^{@}$ & 13.50 & 13.50 & 15.00 & 15.00 & 15.00 & - & - & - & - & - & 14.40 \\
\hline
\end{tabular}

@ - Solvent water mixture (1:1); Observations are mean of three replications; within column means followed by the same letter are not differ significantly using LSD test, $(\mathrm{P}=0.05)$ levels. Values in parenthesis are arcsine transformed values; ${ }^{\wedge}$ Observed on $1,3,5,7,10,14$ and 21 days after treatment $(\mathrm{DAT})$. 
Table.5 Physical compatibility test of hexane crude extracts of Lantana leaves with Neem oil and NSKE

\begin{tabular}{|c|c|c|c|c|c|}
\hline \multirow{2}{*}{$\begin{array}{l}\text { S. } \\
\text { No. }\end{array}$} & \multirow{2}{*}{ Treatments ( $\%$ Concentration) } & \multirow{2}{*}{$\begin{array}{l}\text { Creaming matter } \\
\text { at } \\
\text { the top }(\mathrm{ml})\end{array}$} & \multirow{2}{*}{$\begin{array}{l}\text { Creaming matter at } \\
\text { the bottom (ml) }\end{array}$} & \multicolumn{2}{|c|}{ Phytotoxicity (\%) } \\
\hline & & & & Cabbage & Cauliflower \\
\hline 1 & Lantana hexane crude extracts 1.00 & - & - & - & - \\
\hline 2 & Lantana hexane crude extracts 2.00 & - & - & - & - \\
\hline 3 & Lantana hexane crude extracts 4.00 & - & - & - & - \\
\hline 4 & Lantana hexane crude extracts 6.00 & - & - & - & - \\
\hline 5 & Lantana hexane crude extracts 8.00 & - & 0.10 & 2.00 & 33.00 \\
\hline 6 & Lantana hexane crude extracts 10.00 & - & 0.20 & 9.30 & 49.50 \\
\hline 7 & $\begin{array}{l}\text { Lantana hexane crude extracts } 6.00+ \\
\text { Neem oil } 3.00\end{array}$ & 0.20 & - & - & - \\
\hline 8 & $\begin{array}{l}\text { Lantana hexane crude extracts } 6.00+ \\
\text { NSKE } 5.00\end{array}$ & 0.10 & - & - & - \\
\hline 9 & $\begin{array}{l}\text { Lantana hexane crude extracts } 6.00+ \\
\text { APSA } 80(0.30 \mathrm{ml})\end{array}$ & - & - & - & - \\
\hline 10 & $\begin{array}{l}\text { Lantana hexane crude extracts } 6.00+ \\
\text { APSA } 80(0.30 \mathrm{ml})+\text { Hexane }\end{array}$ & - & - & - & - \\
\hline
\end{tabular}


Table.6 Leaf injury was assessed by visual rating in 0-10 scale

\begin{tabular}{cc}
\hline Rating & Phytotoxicity (\%) \\
\hline 0 & No phytotoxicity \\
1 & $1-10$ \\
2 & $11-20$ \\
3 & $21-30$ \\
4 & $31-40$ \\
5 & $41-50$ \\
6 & $51-60$ \\
7 & $61-70$ \\
8 & $71-80$ \\
9 & $81-90$ \\
10 & $91-100$ \\
\hline
\end{tabular}

It is due to L. camara may produce and release several types of secondary metabolites, including phenolic acids, flavonoids, terpenes and terpenoids. Among these secondary metabolites, some are known allelochemicals inhibiting the growth of other organisms and weeds (Mersie and Singh, 1987; Singh et al., 1989; Sharma et al., 2000).

In conclusion, results showed $6 \%$ is highly toxic to $\mathrm{P}$. xylostellalarvae after ingestion or contact on diamondback moth larvae. Its effectiveness under pot culture persisted up to 14days after treatment, and the residues will likely last 10-14 days. We select the 6\% was very effective against $P$. xylostella under the pot culture experiment. Lantana crude extracts of $6 \%$ evaluated in field trialsrepresent valuable new tools as a repellent that providegrowers with alternatives to currently used insecticides. It is expected that hexane crude extracts will play an important role in push pull strategies with its novel mode of action, quick cessation of feeding, persistence under field conditions and compatibility with natural enemies.

\section{Acknowledgement}

We are very grateful to Department of Agricultural Entomology, Tamil Nadu agricultural University and University Grants
Commission (UGC) for generous financial and good support (Project Number: 56379/2013-14) for doctoral research work.

\section{References}

Badii, K.B., Adarkwah, C. and Nboyine, J.A. 2013. Insecticide use in cabbage pest management in tamale metropolis of Ghana. Greener Journal of Agricultural Sciences, 3: 403-411.

Cloyd, R.A. 2012. Indirect Effects of Pesticides on Natural Enemies, Pesticides - Advances in Chemical and Botanical Pesticides. In: Soundararajan, R. P. (eds), Available: http://www.intechopen.com/books/pesti cides-advances-in-chemical-andbotanical-pesticides/ indirect effects- of -pesticides-on-natural-enemies. p. 120

Dash, A.N., Senapati, B and Samalo, A.P. 1995. Persistent Toxicity of Neem Derivatives to Green Leafhopper, Nephotettix virescens Distant (Cicadellidae: Homoptera) Indian Journal of Plant Protection, 23(1): 4346.

DeBach, P. 1974. Biological control by natural enemies. In: Rosen, D. (ed.) Cambridge, Cambridge University Press, UK. pp. 1-113 
FAOSTAT.2011. Food and Agriculture Organization Statistical Database. Available: http://www.cropnutrition/crops/vegetable-brassica/keyfacts/world-pro.aspx.

Furlong, M.J., Wright, D.J. and Dosdall, L.M. 2013. Diamondback moth ecology and management: problems progress and prospects. Annual Review of Entomology, 58: 517-541.

Harcourt, D.G. 1954. The biology and ecology of the diamondback moth, Plutella maculipennis, Curtis, in Eastern Ontario. PhD thesis. Cornell University., Ithaca, N.Y. p. 107.

Haynes, K.F.1988.Sublethal effects of neurotoxic insecticides on insect behaviour. Annual Review of Entomology, 33: 149-168.

Indian Horticulture Data Base. 2013. National Horticulture Board, Ministry of Agriculture, GOI - 85, Institutional Area, Sector-18, Gurgaon, India. www.nhb.gov.in.

Johnson, R.M., Pollock, H.S. and Berenbaum, M.R. 2009. Synergistic interactions between in-hive miticides in Apis mellifera. Journal of Economy Entomol, 102: 474-79.

Kong, C.H., Wang, P., Zhang, C.X., Zhang, M.X and $\mathrm{Hu}, \mathrm{F}$. 2005. Herbicidal potential of allelochemicals from Lantana camara against Eichhornia crassipes and the alga Microcystis aeruginosa. Weed Research, 46: 290295.

Krishna Moorthy P.N., Krishna Kumar, N.K. and Edward Raja, M. 2002. Souvenir of 4th World Neem Conference. Mumbai, November 27-30: 60-67.

Liu, T.X., Sparks, A.N. and Chen, J.R.W. 2003. Toxicity, persistence and efficacy of indoxacarb and two other insecticides on Plutella xylostella (Lepidoptera: Plutellidae) immatures in cabbage.
International Journal of Pest Management, 49(3): 235-241.

Liu, Y.Q., Shi, Z.H., Zalucki, M.P. and Liu, S. 2014. Conservation biological control and IPM practices in Brassica vegetable crops in China. Biological Control,

68: 37-46.

Mersie, W and Singh, M. 1987. Allelopathic effect of Lantana on some agronomic crops and weeds. Plant and Soil, 98: $25-30$.

Mohamad, R.B. and Ismail, M.Y. 1988. Persistence of insecticides against larvae of Plutella xylostella L. Insect Scienc and Applications, 9(1): 109-112.

Santos, V.C., de Siqueira, H.A., da Silva, J.E. and de Farias, M.J. 2011. Insecticide resistance in populations of the diamondback moth, Plutella xylostella (L.) (Lepidoptera: Plutellidae), from the state of Pernambuco, Brazil. Neotropical Entomology, 40: 264-270.

Sarfraz, M. and Keddie, B.A. 2005. Conserving the efficacy of insecticides against Plutella xylostella (L.) (Lepidoptera: Plutellidae). Journal of Applied Entomology, 129: 149-157.

Sayyed, A.H., Omar, D. and Wright, D.J. 2004. Genetics of spinosad resistance in a multi-resistance field selected population of Plutella xylostella L. Pest Management Science, 60: 827-832.

Sharma, O.P., Singh, A and Sharma, S. 2000. Levels of lantadenes, bioactive pentacyclic triterpenoids in young and mature leaves of Lantana camara var. aculeate. Fitoterapia, 71: 487-491.

Singh, M., Tamma, R.V and Nigg, H.N.1989. HPLC identification of allelopathic compounds from Lantana camara. Journal of Chemical Ecology, 15: 8189.

Sharma, S., Senrung, A. and Singh, A.K. 2014. Toxic effect of neem, Azadirachta indica (A. Juss) foliage extracts against diamondback moth (DBM), Plutella 
xylostella (L.) (Lepidoptera: Northern Thailand, Bangkok, Thailand, Plutellidae). Journal of Biopesticide, 7:99-105.

Sukthamrong. A., Subhadrabandhu, S., Reutrakul, V., Sagwansupyakorn, C., Chandra-prasong, C. and Tuntiwachuttikul, C. 1981. Research on identification and production of diosgenin produce plant for Opium popy substitute in the high land of Zhao, J.Z., Li, Y.X., Collins, H.L.,

Kasetsart University. p.31. Gusukuma, M.L., Mau, R.F.L. and Thompson, G.D. 2002. Monitoring and characterization of diamond back moth (Lepidoptera: Plutellidae) resistance to spinosad. Journal of Economic Entomology, 95: 430-436.

\section{How to cite this article:}

Thanavendan, G. and Kennedy, J.S. 2017. Persistence, Relative Efficacy and Phytotoxicity of Lantana camara var. Aculeata (L.) Moldenke Leaf Crude Extracts in Hexane against Plutella xylostella L. in Cruciferous Vegetables. Int.J.Curr.Microbiol.App.Sci. 6(6): 3201-3212. doi: https://doi.org/10.20546/ijcmas.2017.606.377 